\title{
Editorial
}

\section{The uncomfortable comfort zone}

\author{
Carlos Renato Zacharias \\ International Journal of High Dilution Research - Editor-in-Chief
}

\begin{abstract}
Whenever we achieve the satisfaction of our expectations and anxiety dissolves, we feel as if we were in a comfort zone - safe, complete, free from risks and in peace with ourselves. We might even have a little taste of heaven when we feel that we have fulfilled our duty. And as a fact, scientists are entitled to this kind of reward... but not for too long! In science, to enter a comfort zone can be as pleasant as dangerous. On one hand we may have a safe ground available to develop new modes of reasoning, protocols and theories. And on the other, we may stay stuck in a conventional but fragile ground, missing opportunities to reveal novel secrets or to address edge issues.
\end{abstract}

Fundamental research in High Dilutions is at risk of entering such a state. Recently, nanostructures were found in serially diluted and agitated fluids, which not only raised our expectations as to a novel conceptual framework able to explain the HD phenomenon, but also led us back to mainstream science and the most innovative experimental technology. This has more far reaching consequences: if nanostructures form in HD, then Avogadro's limit becomes senseless, and the long unexplained clinical and pharmacological properties of HD might be finally elucidated, new experiments may be designed, new theories postulated, and so forth. Shortly, once persuaded we hit the gold vein, we might celebrate this new era in the history of HD research and claim our duly place in mainstream science.

The expectation of explaining HD and homeopathy out of nanostructures is not shared by our entire community. Some still prefer to associate this phenomenon with immaterial factors, like the putative vital energy or some still unknown property of matter. Others simply refuse such structural hypotheses because they do not fulfill basic requirements to explain the specificity of homeopathic medicines.

Despite personal opinions or beliefs, the presence of nanostructures is, indeed, a consistent hypothesis and experimental evidences might accumulate and make it ever sounder. It is reasonable to posit that the energy released by mechanical agitation induces cavitation in the liquid phase and thus triggers nonlinear effects resulting in new molecular rearrangements. To follow this structural track means to keep us inside mainstream science, since we deal with sound theory, might perform modern experiments and collect data fit to be interpreted within the framework the current data.

Nanostructures were identified only very recently and they had not been yet fully investigated. Countless data must still be collected, results organized, and eventually, new methods developed. The required tests are extremely complex, whereas the HD community suffers from a chronic and severe lack of human and material resources. In spite of such shortcomings, slowly and gradually we might become able to formulate a consistent theory both supporting and being supported by experimental evidence. This, as a fact, is the natural time of science. Adjusting for scale, we might judge we are following a track similar to the one that leads us to the atomic and the electromagnetic models, which do not started at quantum mechanics or Maxwell's equations, but from isolated experimental observations. 
However, several issues still require thorough investigation: Are such nanostructures truly generated by serial dilution and agitation? Do they actually derive from the starting-material? Are nanostructures altered along serial dilution and agitation, as the clinical results predict? Even assuming that the presence of nanostructures in HD has the full weight of evidence, a very long road must still be travelled before we might be able to attribute biological activity to them. Also, since historically the samples have always been prepared in glass vials, we still must rule out the possibility of them being experimental artifacts due to the presence of contaminants, or results of the drying procedures required to prepare samples for experimental analysis.

Few years ago, I challenged some colleagues who were utterly persuaded of the physical-chemical nature of HD to cite one single result correlating the identified physical-chemical properties of HDs and the biological activity of such preparations. There is no need to say that I'm still waiting for an answer. And the same applies to nanostructures: they will not represent an answer until they had not been correlated with biological activity and their mechanism is not shown to fully agree with the well-known characteristics of homeopathic medications, namely dependence on the starting-material and different behavior according to the level of dilution and agitation.

The experimental and conceptual difficulties, the lack of a critical mass of researches, and the pressure exerted by the university and scientific milieu on the few existing ones might make us loose from sight the fact that the latest positive experiment is never the ultimate explanation of the HD phenomenon. Conversely, conflict and debate are systematically more fruitful in science than consensus and convergence.

The community of HD researchers seems to have entered a new comfort zone when nanostructures were found in HD. Nanostructures have been raised to the level of ultimate evidence doing away with Avogadro's limit and leading homeopathy and HD research into mainstream science. We really should enjoy this moment and collect more information about the HD phenomenon. However, we must be wary to not fall into a trap.

\section{(c)) BY-NC-ND Licensed to GIRI}

How to cite this article: Zacharias, CR. The uncomfortable comfort zone [editorial]. Int J High Dilution Res [online]. 2012 [cited YYYY Month dd]; 11(38): 01-02. Available from: http://www.feg.unesp.br/ ojs/index.php/ijhdr/article/view/550/558 